\title{
Enfermedad de Behçet frente a la enfermedad inflamatoria intestinal. Diferencias en la presentación clínica
}

\section{Behçet's disease compared with inflammatory bowel disease. Differences in their clinical presentation}

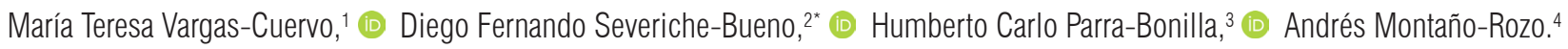

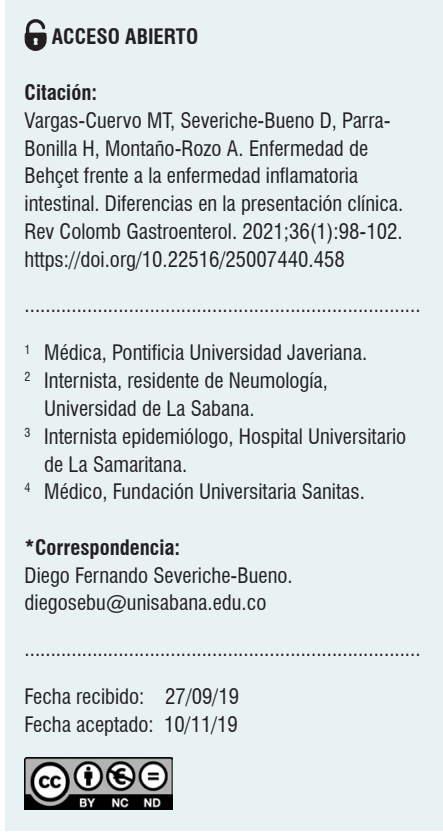

\begin{abstract}
Resumen
La enfermedad de Behçet es una enfermedad poco frecuente con un compromiso sistémico cuyas principales manifestaciones abarcan el sistema gastrointestinal, neurológico, vascular y articular, además de la piel y los ojos. Por su compromiso multisistémico, es frecuente confundirlo con otras entidades como la enfermedad inflamatoria intestinal, con la cual comparte gran parte de su presentación clínica, por lo que se describe un caso clínico y se aborda una revision narrativa desde el punto de vista epidemiológico de las manifestaciones clínicas con el fin de tener un mayor conocimiento para identificarla a tiempo.
\end{abstract}

Palabras clave

Enfermedad de Behçet, colitis ulcerativa, enfermedad inflamatoria intestinal.

\begin{abstract}
Behçet's disease is a rare disease with systemic involvement whose main manifestations include the gastrointestinal, neurological, vascular, and articular systems, as well as the skin and eyes. Due to its multisystemic nature, it is often mistaken for other entities such as inflammatory bowel disease since they share multiple characteristics in their clinical presentation. A clinical case is described, and an epidemiological review of clinical manifestations is discussed to have better knowledge that allow identifying it on time.
\end{abstract}

\section{Keywords}

Behçet's disease, Ulcerative colitis, Inflammatory bowel disease.

\section{INTRODUCCIÓN}

La enfermedad de Behçet es una vasculitis infrecuente de etiología desconocida que se caracteriza por la presencia de aftas orales recurrentes y cualquiera de sus manifestaciones sistémicas que incluyen alteraciones a nivel del tracto gastrointestinal $(1,2)$.

La presentación clínica más común es la presencia de úlceras mucocutáneas recurrentes; sin embargo, hasta en un $10 \%$ de los pacientes se presentan manifestaciones gastrointesti- nales que pueden ser los primeros síntomas de la enfermedad $y$, dado lo heterogéneo que puede ser su presentación clínica, se puede volver un reto diagnóstico diferenciarla de la enfermedad inflamatoria intestinal (EII) $(1,2)$.

Por lo anterior, presentamos un caso de enfermedad de Behçet para discutir mediante una revisión narrativa las diferencias que presenta con respecto a la enfermedad inflamatoria intestinal desde el punto de vista epidemiológico de las manifestaciones clínicas para brindar una luz al momento de diferenciar estas dos entidades. 


\section{CASO CLÍNICO}

Se trata de una paciente de 61 años con cuadro clínico de 6 meses de evolución consistente en la aparición de lesiones eritematosas no pruriginosas inicialmente en ambas rodillas, con posterior aparición en los muslos, abdomen, región lumbar y glútea; las cuales eran episódicas y migrantes. Asociado a esto, refirió deposiciones líquidas amarillentas que aumentaron en frecuencia, además de presentar rectorragia, picos febriles y sangrado vaginal del mismo tiempo de evolución; síntomas que se exacerbaron en las dos semanas previas a la consulta. También refirió úlceras orales recurrentes de 4 meses de evolución y deterioro de su clase funcional.

Como antecedente, comentó que a los dos meses de presentar el cuadro le habían diagnosticado colitis ulcerativa $(\mathrm{CU})$, por lo que estaba en manejo ambulatorio con mesalazina oral. Según lo referido por la paciente, el diagnóstico se realizó a través de una colonoscopia; sin embargo, no contaba con el estudio patológico a su disposición. Al examen físico se documentó una úlcera de fondo gris y un halo eritematoso periférico en la mucosa del labio inferior y 2 úlceras de fondo gris en la región interna de los labios menores en la vulva. Sumado a esto, se observaron pápulas y placas eritematosas de ambos muslos. No se documentaron lesiones oculares ni hallazgos a nivel articular.

Fue valorada por medicina interna y gastroenterología considerando una exacerbación de su CU (Truelove 19) con manifestaciones extraintestinales. Se realizó una colonoscopia y se encontró a nivel del sigmoides una mucosa edematizada, friable, ulcerada, con pérdida de patrón glandular y vascular y formación de pseudopólipos. Sin embargo, durante el curso de su hospitalización la paciente presentó cefalea hemicraneal, por lo que dentro de los estudios realizados se diagnosticó un aneurisma lobulado de la arteria cerebral media derecha. Sumado a esto, también refirió dolor torácico documentándose posteriormente una cardiomiopatía con fracción de eyección del ventrículo izquierdo (FEVI) del $38 \%$ con arterias coronarias sanas.

Por su evolución clínica se revaloró el enfoque del caso y se planteó el diagnóstico diferencial de una enfermedad de Behçet, por lo que se ordenó el HLA-B51, que fue negativo, y una biopsia de piel. Durante su evolución, se recibió el reporte de patología del colon que describió una úlcera con tejido de granulación, infiltrado linfoplasmocitario y neutrofílico, con cambios epiteliales reactivos compatible con una colitis crónica activa. El reporte de la biopsia de las úlceras en la mucosa oral y vaginal, describió la presencia de úlceras de carácter no específico con una grave inflamación aguda y crónica. La biopsia de piel reportó una dermatitis de interfase.

Ante la dificultad clínica del caso, se realizó una junta entre dermatología, medicina interna y gastroenterología, en la que se planteó que era más probable el diagnóstico de enfermedad de Behçet, dado el compromiso multisistémico de la paciente y los hallazgos clínicos y paraclínicos con lo que marcaba 6 puntos en los criterios internacionales para la enfermedad de Behçet (ICBD). Con respecto a la evolución de la paciente, se llevó a una corrección quirúrgica del aneurisma cerebral, se le inició el manejo de su insuficiencia cardíaca y se continuó el manejo inmunomodulador con prednisolona, colchicina, azatioprina y mesalazina con una adecuada evolución clínica, y posterior resolución de la diarrea, las lesiones en piel y las lesiones en la mucosa, con su consecuente egreso. En los seguimientos por consulta externa de reumatología se observó remisión de la enfermedad marcando 0 puntos de los criterios de ICBD.

\section{DISCUSIÓN}

\section{Epidemiología general}

Dentro de las enfermedades inflamatorias intestinales, la $\mathrm{CU}$ es el desorden inflamatorio gastrointestinal con más manifestaciones extraintestinales similares a las presentadas en la enfermedad de Behçet (1). Su incidencia varía según la región, aunque es mayor en Inglaterra, Norteamérica y el norte de Europa, con predominio en los individuos de 15 a 29 años sin diferencias entre sexos $(1,2)$. En contraste, la enfermedad de Behçet se da principalmente alrededor de la ruta de seda, en especial en Turquía, y tiende a afectar a adultos jóvenes entre los 20-40 años (1).

\section{Epidemiologia de hallazgos clínicos}

Las manifestaciones oculares se pueden observar en un $25 \%$ $75 \%$ de los pacientes con enfermedad de Behçet (3). La manifestación ocular más frecuente es la uveítis, la cual se presenta de manera bilateral y episódica (4). Así mismo, el $3 \%$ de los pacientes con enfermedad de Behçet presenta úlceras conjuntivales $(5,6)$. En cambio, en los pacientes con EII el compromiso ocular más común es la epiescleritis, que ocurre en el $2 \%$ a $5 \%$ de los pacientes y se exacerba cuando existe actividad de la enfermedad (7). En cuanto a la uveítis, puede ocurrir en un 0,5 a $3 \%$ de los pacientes con EII, la cual ocurre con más frecuencia en mujeres (8).

La manifestación articular más frecuente en pacientes con enfermedad de Behçet, que se observa en el $50 \%$ de los pacientes, es la artritis no erosiva y asimétrica (9). En cambio, la artritis es la manifestación extraintestinal más común de la EII. Un $3 \%-10 \%$ de los pacientes con EII pueden tener espondilitis anquilosante. La artritis en la EII se diferencia con la enfermedad de Behçet por la presencia de sacroileítis y entesitis (10-12). 
En cuanto a las manifestaciones mucocutáneas, en la enfermedad de Behçet hasta el $50 \%$ de los pacientes tiene eritema nodoso o lesiones similares (13). Es un escenario similar con respecto a la EII, en la que un $40 \%$ de los pacientes puede presentar eritema nodoso como manifestación extraintestinal (14). Adicionalmente, en la enfermedad de Behçet, la mayoría inicia con aftas orales extensas y múltiples en lengua, labios y mucosa oral, por lo que cursan con estomatitis aftosa recurrente, con una frecuencia que se estima entre el $97 \%$ y el $100 \%$. Lo anterior contrasta con la EII, en la que la estomatitis aftosa recurrente tiene una incidencia del $10 \%$ en pacientes con enfermedad de Crohn (EC) y del $4 \%$ en la CU $(14,15)$. Con respecto al compromiso de la mucosa genital en la enfermedad de Behçet, las úlceras tienden a dejar cicatriz, se presentan en un 56,7 \%-97 \% de los casos y es común su presencia en la vulva, la vagina y el cérvix (16). En contraste, en la EII no se presenta compromiso de la mucosa genital y solo se ha reportado compromiso vulvar que se manifiesta como eritema, edema, dolor y prurito de la vulva, del cual solo se han descrito 200 casos en pacientes con EC (17).

Con respecto a la miocardiopatía, esta se puede observar en el $6 \%$ de los pacientes con enfermedad de Behçet e involucra a cualquiera de las capas del corazón. En la EII, en una cohorte danesa con 15572 pacientes se reportaron apenas 6 casos de miocarditis y los estudios epidemiológicos estimaron una incidencia de miocardiopatía de 8,3 para EC y 2,6 para CU; en esta última un porcentaje importante se dio por toxicidad a la mesalazina, lo cual resalta el hecho de que es más común el compromiso cardíaco en la enfermedad de Behçet que en la EII (18).

El compromiso vascular es una de las principales causas de morbimortalidad en la enfermedad de Behçet, con una prevalencia de $14,3 \%$, y es más común en hombres. Las arterias comúnmente afectadas son las carótidas, las pulmonares, las ilíacas y la aorta; el compromiso de las arterias renales y cerebrales es menos frecuente (4). En la EII, un metaanálisis de 2014 encontró un riesgo ligeramente aumentado de accidentes cerebrovasculares (Odds ratio $[\mathrm{OR}]: 1,18)$ con un aumento del riesgo más prominente en mujeres y pacientes jóvenes. También se han visto trombosis venosas cerebrales, que son más frecuentes en los pacientes con CU que en la EC. Lo expuesto anteriormente muestra que la EII tiene una leve asociación con eventos trombóticos (arteriales o venosos) en el sistema nervioso central, mientras que la enfermedad de Behçet tiende a comprometer los grandes vasos además de tener una mayor predisposición a formar aneurismas (19).

Por último, el compromiso gastrointestinal en la enfermedad de Behçet ocurre en aproximadamente el $10 \%$ de los pacientes y varía según la región. Este se puede manifestar con dolor abdominal, diarrea y sangrado, y no es raro que se confunda con la EII. Usualmente compromete la región ileocolónica, especialmente el área ileocecal, pero puede comprometer cualquier región del tracto gastrointestinal. Sin embargo, siempre respeta el recto, a diferencia de la CU, en la que el compromiso del recto es frecuente (20).

En cuanto al diagnóstico de la enfermedad de Behçet, se pueden usar los criterios internacionales (ICBD) (Tabla 1), los criterios japoneses (Tabla 2) o los criterios del grupo de estudio internacional para enfermedad de Behçet (ISG), entre otros criterios diagnósticos descritos (Tabla 3) (2123). Es de resaltar que los tres grupos hacen énfasis en manifestaciones clínicas y no paraclínicas. Con respecto a pruebas como el HLA-B51 y la prueba de patergia, ninguna es especifica ni sensible como para descartar o confirmar la enfermad. Incluso, el rendimiento de la prueba de patergia varía según la etnia o la localización geográfica: tiene mayor rendimiento en Oriente Medio y un rendimiento de apenas $5 \%$ en los pacientes caucásicos $(24,25)$.

Tabla 1. Criterios internacionales para la enfermedad de Behçet (ICBD) (21)

\begin{tabular}{lc|}
\multicolumn{1}{c}{ Hallazgos } & Puntos \\
\hline Úlceras aftosas orales & 2 puntos \\
Úlceras aftosas genitales & \\
Manifestaciones oculares & \\
Lesiones en piel & 1 punto \\
Manifestaciones neurológicas & \\
Manifestaciones vasculares & \\
Prueba de patergia positiva &
\end{tabular}

Diagnóstico con $\geq 4$ puntos

Con respecto a similitudes en su fisiopatología, se ha visto que en el cromosoma 1p31.1 las regiones IL23R e IL12Rb2 cuando se expresan favorecen una predisposición a desarrollar la enfermedad de Behçet. Estas mismas regiones se han visto involucradas en los pacientes con EII, cuyas variantes de estas regiones se han asociado con el desarrollo de EII. Adicionalmente, ambas enfermedades tienen una actividad elevada de Th1, Th17, CD4+ y CD8+, lo cual sugiere un rol del sistema innato y adaptativo del sistema inmune en su fisiopatología (1).

\section{CONCLUSIONES}

Debido a que la enfermedad de Behçet comparte muchas características con la EII, suele convertirse en un reto diagnóstico para cualquier médico. Por esto, es importante 
Tabla 2. Criterios japoneses (22)

\section{Criterios mayores}

Úlceras aftosas recurrentes en la mucosa oral

Lesiones en la piel

Lesiones oculares

Úlceras genitales

Criterios menores

Artritis sin deformidad o anquilosis

Lesiones gastrointestinales caracterizadas por úlceras ileocecales

Epididimitis

Lesiones vasculares

Síntomas del SNC

Prueba de patergia positiva

Prueba de patergia cutánea con estreptococos muertos

HLA-B51 positiva

Laboratorios consistentes con una respuesta inflamatoria

\section{Clasificación}

Completa $\quad 4$ criterios mayores presentes

Incompleta 3 mayores

2 mayores y 2 menores

Síntomas oculares típicos recurrentes más un criterio mayor o dos menores

Sospecha $\leq 2$ sintomas mayores

Criterios menores que recurren o aumentan en gravedad
Tabla 3. Criterios del grupo de estudio internacional para enfermedad de Behçet (ISG) (23)

Ulceración oral recurrente: afta mayor o menor o ulceración herpetiforme observada por el médico o paciente, la cual ha recurrido al menos 3 veces en un año

Más 2 de los siguientes:

- Ulceración genital recurrente: ulceración o cicatriz aftosa observada por el médico o pacientes

- Lesiones oculares: uveítis anterior o posterior o células en vítreo con examen de lámpara de hendidura o vasculitis retinal observada por el oftalmólogo

- Lesiones cutáneas: eritema nodoso observado por el médico o paciente, pseudofoliculitis o lesiones papulopustulosas 0 nódulos acneiformes observados por el médico o paciente en posadolescentes sin tratamiento cortical

Prueba de patergia positiva

reconocer aquellas manifestaciones clínicas más frecuentes en cada una de estas entidades para orientar el diagnóstico. La presencia de úlceras en la mucosa genital, el compromiso cardíaco, el respeto del recto y el compromiso de grandes vasos ayuda a inclinar la balanza hacia la enfermedad de Behçet en aquellos pacientes que cursan con síntomas gastrointestinales y no hay claridad si se trata de una EII.

Debido a que todavía no existe un examen patognomónico que oriente de manera precisa su diagnóstico, es importante que artículos como este estimulen la investigación en esta patología para encontrar nuevos métodos diagnósticos que ayuden a una aproximación diagnóstica más rápida y eficiente. En ambas patologías la historia clínica y el examen físico siguen teniendo un rol importante y aclaratorio.

\section{REFERENCIAS}

1. Valenti S, Gallizzi R, De Vivo D, Romano C. Intestinal Behçet and Crohn's disease: two sides of the same coin. Pediatr Rheumatol Online J. 2017;15(1):33. https://doi. org/10.1186/s12969-017-0162-4

2. Yazısız V. Similarities and differences between Bechet's disease and Crohn's disease. World J Gastrointest Pathophysiol. 2014;5(3):228-38. https://doi. org/10.4291/wjgp.v5.i3.228

3. Nussenblatt RB. Uveitis in Bechet's disease. Int Rev Immunol. 1997;14(1):67-79. https://doi. org/10.3109/08830189709116845
4. Seyahi E, Melikoglu M, Yazici H. Clinical features and diagnosis of Behcet's syndrome. Int J Adv Rheumatol. 2007;5(1):8-13.

5. Matsuo T, Itami $M$, Nakagawa $H$, Nagayama $M$. The incidence and pathology of conjunctival ulceration in Bechet's syndrome. Br J Ophthalmol. 2002;86(2):140-3. https:// doi.org/10.1136/bjo.86.2.140

6. Zamir E, Bodaghi B, Tugal-Tutkun I, See RF, Charlotte F, Wang RC, Wechsler B, LeHoang P, Anteby I, Rao NA. Conjunctival ulcers in Behçet's disease. Ophthalmology. 
2003;110(6):1137-41. https://doi.org/10.1016/S01616420(03)00265-3

7. Petrelli EA, McKinley M, Troncale FJ. Ocular manifestations of inflammatory bowel disease. Ann Ophthalmol. 1982;14(4):356-60.

8. Lyons JL, Rosenbaum JT. Uveitis associated with inflammatory bowel disease compared with uveitis associated with spondyloarthropathy. Arch Ophthalmol. 1997;115(1):61-4. https://doi.org/10.1001/archopht.1997.01100150063010

9. Kim HA, Choi KW, Song YW. Arthropathy in Bechet's disease. Scand J Rheumatol. 1997;26(2):125-9. https:// doi.org/10.3109/03009749709115831

10. Biggioggero M, Crotti C, Becciolini A, Miserocchi E, Favalli EG. The Management of Acute Anterior Uveitis Complicating Spondyloarthritis: Present and Future. Biomed Res Int. 2018;2018:9460187. https://doi. org/10.1155/2018/9460187

11. Ehrenfeld M. Spondyloarthropathies. Best Pract Res Clin Rheumatol. 2012;26(1):135-45. https://doi. org/10.1016/j.berh.2012.01.002

12. Holden W, Orchard T, Wordsworth P. Enteropathic arthritis. Rheum Dis Clin North Am. 2003;29(3):513-30, viii. https://doi.org/10.1016/s0889-857x(03)00043-7

13. Requena L, Requena C. Erythema nodosum. Dermatol Online J. 2002;8(1):4.

14. Huang BL, Chandra S, Shih DQ. Skin manifestations of inflammatory bowel disease. Front Physiol. 2012;3:13. https://doi.org/10.3389/fphys.2012.00013

15. Lee ES, Bang D, Lee S. Dermatologic manifestation of Bechet's disease. Yonsei Med J. 1997;38(6):380-9. https:// doi.org/10.3349/ymj.1997.38.6.380

16. Ideguchi H, Suda A, Takeno M, Ueda A, Ohno S, Ishigatsubo Y. Behçet disease: evolution of clinical manifestations. Medicine (Baltimore). 2011;90(2):125-132. https://doi.org/10.1097/MD.0b013e318211bf28

17. Abboud ME, Frasure SE. Vulvar inflammation as a manifestation of Crohn's disease. World J Emerg
Med. 2017;8(4):305-307. https://doi.org/10.5847/ wjem.j.1920-8642.2017.04.011

18. Katsanos $\mathrm{KH}$, Tsianos EV. The heartin inflammatory bowel disease. Ann Gastroenterol. 2002;15(2):124-133.

19. Dolapcioglu C, Dolapcioglu H. Structural brain lesions in inflammatory bowel disease. World J Gastrointest Pathophysiol. 2015;6(4):124-30. https://doi. org/10.4291/wjgp.v6.i4.124

20. Hatemi I, Hatemi G, Çelik AF. Gastrointestinal Involvement in Behçet Disease. Rheum Dis Clin North Am. 2018;44(1):45-64. https://doi.org/10.1016/j. rdc.2017.09.007

21. International Team for the Revision of the International Criteria for Bechet's Disease (ITR-ICBD). The International Criteria for Bechet's Disease (ICBD): a collaborative study of 27 countries on the sensitivity and specificity of the new criteria. J Eur Acad Dermatol Venereol. 2014;28(3):338-47. https://doi.org/10.1111/jdv.12107

22. Suzuki Kurokawa M, Suzuki N. Behcet $>$ s disease. Clin Exp Med. 2004;4(1):10-20. https://doi.org/10.1007/s10238004-0033-4

23. Scherrer MAR, Rocha VB, Garcia LC. Bechet's disease: review with emphasis on dermatological aspects. An Bras Dermatol. 2017;92(4):452-464. https://doi.org/10.1590/ abd1806-4841.20177359

24. Greco A, De Virgilio A, Ralli M, Ciofalo A, Mancini $\mathrm{P}$, Attanasio G, de Vincentiis M, Lambiase A. Bechet's disease: New insights into pathophysiology, clinical features and treatment options. Autoimmun Rev. 2018;17(6):567575. https://doi.org/10.1016/j.autrev.2017.12.006

25. Hatemi G, Christensen R, Bang D, Bodaghi B, Celik AF, Fortune F, Gaudric J, Gul A, Kötter I, Leccese P, Mahr A, Moots R, Ozguler Y, Richter J, Saadoun D, Salvarani C, Scuderi F, Sfikakis PP, Siva A, Stanford M, Tugal-Tutkun I, West R, Yurdakul S, Olivieri I, Yazici H. 2018 update of the EULAR recommendations for the management of Bechet's syndrome. Ann Rheum Dis. 2018;77(6):808-818. https:// doi.org/10.1136/annrheumdis-2018-213225 